Classification

Physics Abstracts

85.25-J $-44.10+\mathrm{i}-83.20-\mathrm{d}$

\title{
Etude théorique et expérimentale d'un processus thermique dans un limiteur supraconducteur de courant
}

\author{
J. Lévêque $\left({ }^{1}\right)$, D. Netter $\left({ }^{1}\right)$, A. Rezzoug $\left({ }^{1}\right)$ et J.P. Caron $\left({ }^{2}\right)$ \\ ( $\left.{ }^{1}\right)$ Groupe de Recherche en Electrotechnique et Electronique de Nancy, Université \\ Henri Poincaré, BP 239, 54506 Vandœuvre-lès-Nancy Cedex, France \\ $\left({ }^{2}\right)$ ENSEM, 2 avenue de la forêt de Haye, 54500 Vandœuvre-lès-Nancy, France \\ (Reçu le 5 àril 1995, révisé le 19 Juillet 1995, accepté le 8 septembre 1995)
}

\begin{abstract}
Résumé. - L'accroissement des courants de court-circuit dans les réseaux électriques a ravivé l'intérêt pour les limiteurs supraconducteurs de courant depuis la mise au point en 1983 de fil supraconducteur à faibles pertes en régime variable. Le principe de fonctionnement de ces limiteurs est fondé sur la transition vers l'état normal du matériau supraconducteur. Les modèles simplifiés s'avèrent insuffisants pour l'étude de la transition, une méthode de résolution numérique du problème électrothermique est donc faite. Les résultats des simulations sont comparés à ceux d'expérimentation puis deux études, l'une de facteurs influant sur la propagation de la transition, l'autre du type de court-circuit, sont menées.
\end{abstract}

\begin{abstract}
The increasing of fault current level in electrical networks leads to a new interest for superconducting current limiters since 1983 when an ac wire with low losses has been developed. They are based on the natural transition from the superconducting state to the normal resistive state. In order to study the transition usual models are not sufficient. This paper deals with a numerical resolution of the electro-thermical coupled problem. Computed and experimental results are favorably compared. Some factors which affect the transition as well as the influence of the starting instant are studied.
\end{abstract}

\section{Nomenclature}

$C_{\mathbf{P}}(\mathrm{J} / \mathrm{Kg} \mathrm{K})$ chaleur spécifique,

$\delta l(\mathrm{~m}) \quad$ longueur élémentaire de conducteur,

$\delta t(\mathrm{~s}) \quad$ durée du défaut,

$\Delta H(\mathrm{~J} / \mathrm{Kg}) \quad$ Variation d'enthalpie du supraconducteur,

$\Delta t(\mathrm{~s}) \quad$ pas de temps,

$f \quad$ coefficient de "current sharing",

$h_{x}(\mathrm{~m}) \quad$ pas spatial suivant l'axe $X$,

$h_{y}(\mathrm{~m}) \quad$ pas spatial suivant l'axe $Y$,

$H(\mathrm{~J} / \mathrm{Kg}) \quad$ enthalpie du supraconducteur,

$I$ (A) courant, 


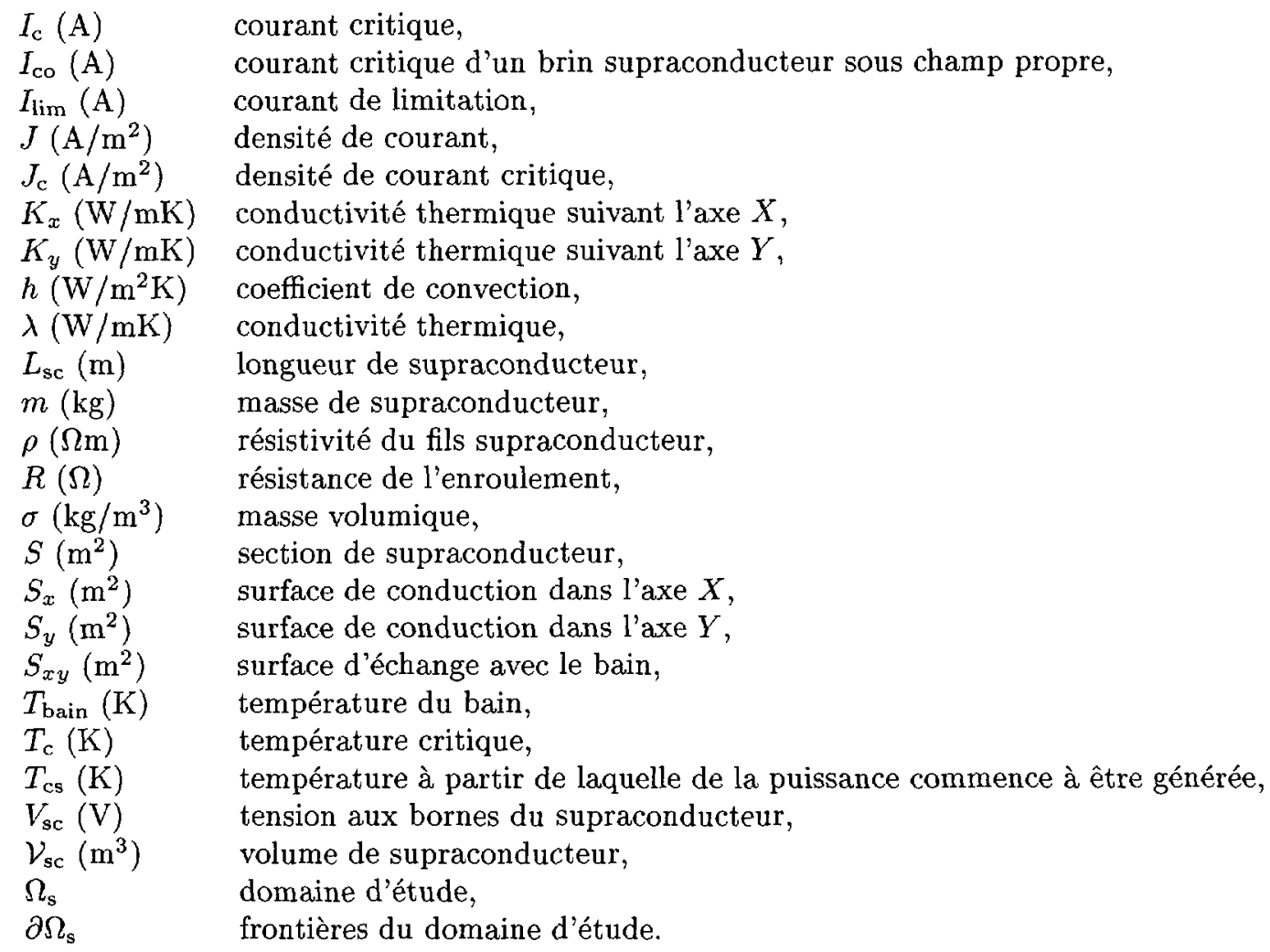

\section{Introduction}

Les valeurs des courants de court-circuit dans les réseaux électriques n'ont cessé d'augmenter atteignant par exemple dans des sous-stations du continent Nord-américain des valeurs de $100 \mathrm{kA}$. Même, en deçà de ces valeurs, les courants de défaut peuvent endommager gravement les installations situées en amont de la charge en raison des efforts électrodynamiques mis en jeu.

Les techniques actuelles sur les réseaux haute tension utilisent des disjoncteurs qui s'ouvrent au passage à zéro du courant. Les plus rapides assurent une coupure en un cycle qui est la durée théorique minimale [1]. Ainsi les composants du réseau doivent supporter les courants de défaut pendant au moins un cycle. Les limiteurs de courants sont des dispositifs particulièrement intéressants puisqu'ils empêchent, en raison de leurs propriétés intrinsèques, l'excursion du courant au-delà d'un seuil et réduisent ainsi les contraintes supportées par les installations électriques. Les distributeurs d'énergie électrique [2] portent une attention toute particulière à ces limiteurs.

L'idée d'un limiteur de courant supraconducteur remonte aux années 1970 [3]. La base de leur fonctionnement est la transition naturelle de l'état supraconducteur vers l'état normal par dépassement du courant critique. Ces limiteurs peuvent être de type résistif (un tel dispositif a été testé avec succès à une échelle significative $[4,5]$ ) ou inductif (des réalisations à petite échelle ont été faites, $[6,7])$.

Si de nombreux modèles ont été étudiés théoriquement et pratiquement, des améliorations significatives peuvent être apportées, cet article contribue à une meilleure compréhension des phénomènes qui accompagnent ces transitions. Des résultats théoriques et pratiques sont présentés. 


\section{Nécessité d'une étude thermique}

La protection de la bobine supraconductrice étant primordiale, la longueur de fils supraconducteur est calculée pour assurer sa protection contre un échauffement excessif après la transition [4-6]. En considérant une transition complète de l'enroulement, en régime adiabatique et isotherme, l'expression de la variation de la température du fil avec le temps est la suivante :

$$
\frac{\mathrm{d} T}{\mathrm{~d} t}=\rho \frac{J^{2}(t)}{C_{\mathrm{P}}(T)}
$$

Après intégration de cette équation la température atteinte par le conducteur est donnée par,

$$
\left(H\left(T_{\max }\right)-H\left(T_{\text {bain }}\right)\right) \mathcal{V}_{\mathrm{sc}}=\frac{1}{R} \int_{0}^{\Delta t} V_{\mathrm{sc}}^{2} \mathrm{~d} t
$$

Ceci permet de calculer la longueur de câble supraconducteur nécessaire à la réalisation du limiteur :

$$
\mathcal{L}_{\mathrm{sc}}=\sqrt{\frac{1}{\rho \Delta H}} \sqrt{\int_{0}^{\Delta t} V_{\mathrm{sc}}^{2} \mathrm{~d} t}
$$

Ce calcul a été effectué dans le cas très restrictif où la transition est massive. En réalité seule une partie du bobinage transite. Il est donc essentiel de connaître la température maximale en tenant compte de la propagation de la chaleur au sein de l'enroulement.

En supposant les paramètres physiques constants, en régime adiabatique la vitesse de propagation d'un front chaud peut être calculée [8] à partir de la relation suivante :

$$
V=\frac{J}{C_{\mathrm{P}}} \sqrt{\frac{\rho \lambda}{T_{\mathrm{s}}-T_{\text {bain }}}} \text { avec } T_{\mathrm{s}}=T_{\mathrm{c}}-\frac{T_{\mathrm{c}}-T_{\text {bain }}}{2} \frac{J}{J_{\mathrm{c}}}
$$

La vitesse mesurée expérimentalement a des valeurs très supérieures à celles calculées par cette méthode [9]. En réalité la vitesse dépend des transferts de chaleur dans chaque spire, entre spires d'une même couche et entre couches, il faut ajouter à cela que les propriétés physiques des matériaux ne sont pas constantes entre $4,2 \mathrm{~K}$ et $300 \mathrm{~K}$.

L'hypothèse d'un régime adiabatique n'est valable que pour une transition rapide et massive. Lorsqu'une faible zone supraconductrice transite, la propagation à la totalité de la bobine est suffisamment lente pour que l'on puisse négliger les termes d'échanges. Aucune des hypothèses faites pour l'étude d'une bobine supraconductrice n'est donc satisfaisante. Une étude thermique plus complète est nécessaire pour dimensionner ce type de bobine.

\section{Modélisation}

Il s'agit de résoudre deux problèmes couplés, le problème thermique, préalablement introduit, et un problème électrique. Le courant décroissant très rapidement dans le limiteur, la puissance dissipée décroît elle aussi. Il est indispensable de calculer la valeur instantanée du courant pour étudier la propagation de la chaleur.

Le modèle doit être suffisant pour appréhender les phénomènes mais assez simple pour permettre de mener jusqu'au bout les calculs des grandeurs recherchées. 


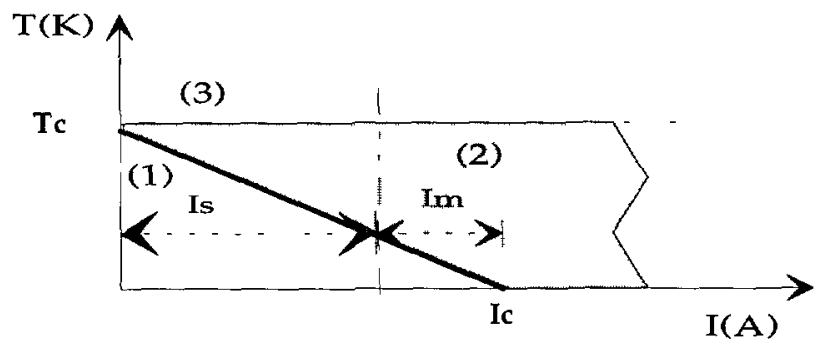

Fig. 1. - Description des zones de "current sharing".

[Current sharing zone description.]

3.1. EquATION THERMiQue. - En considérant un domaine $\Omega_{\mathrm{s}}$ de frontière $\delta \Omega_{\mathrm{s}}$, la formulation mathématique du problème se traduit par l'équation suivante :

$$
\nabla(K(T) \nabla(T))+P(T, I(t))=\sigma C(T) \frac{\partial T}{\partial t} \text { dans } \Omega_{\mathrm{s}}
$$

avec une condition initiale, pour $t=0 T=T_{0}$ dans $\Omega_{\mathrm{s}}$ et une condition sur la frontière,

$$
T=T_{\mathrm{b}} \text { ou } K(T) \frac{\partial T}{\partial n}=0 \text { ou } K(T) \frac{\partial T}{\partial n}=h S\left(T-T_{\mathrm{b}}\right) \text { sur } \partial \Omega_{\mathrm{s}}
$$

$P$ est la somme d'un terme $P_{\mathrm{j}}$ de dissipation d'énergie par effet Joule et d'un terme $P_{\mathrm{c}}$ d'échange de la chaleur lorsque le régime n'est pas adiabatique et qu'il n'est pas inclu dans les conditions aux limites.

Le calcul de $P_{\mathrm{J}}$ dépend de la distribution du courant dans le composite et peut toujours s'écrire sous la forme :

$$
\delta P_{\mathrm{j}}=f \rho \frac{\delta l}{\mathrm{~s}} I^{2}
$$

Pour un fil donné, la variation du courant critique avec la température (Fig. 1), [10,11], peut être représentée par une droite caractérisée par $T_{c}$ et $I_{c}$ pour une valeur d'induction donnée.

Ce modèle permet de prendre en compte la redistribution de courant dans le fil lors de la transition, on distingue alors trois zones de fonctionnement qui sont définies sur la figure 1 et conduisent à trois expressions du coefficient $f$ de l'equation précédente :

(i) Le point est sous la droite critique, le matériau est supraconducteur.

$$
f=0
$$

(ii) Le point se trouve au dessus de la droite critique et $T<T_{\mathrm{c}}$, cette zone est dite de "current sharing" - le courant se répartit dans le composite de telle sorte que les filaments supraconducteurs transportent leur courant critique $I_{\mathrm{s}}$ en gardant leur propriété supraconductrice et que la matrice résistive transporte l'excès de courant $I_{\mathrm{m}}$.

$$
f=\left(T-T_{\mathrm{cs}}\right) /\left(T_{\mathrm{c}}-T_{\mathrm{cs}}\right) \text { avec } T_{\mathrm{cs}}=T_{\mathrm{bain}}+\left(T_{\mathrm{c}}-T_{\mathrm{bain}}\right)\left(1-I / I_{\mathrm{c}}\right)
$$

(iii) Le point est dans la zone $T>T_{\mathrm{c}}$, le matériau n'est plus supraconducteur.

$$
f=1
$$


3.2. EquATION ÉLECTRIQUe. - Le supraconducteur étant parcouru par un courant sinusoïdal, l'équation électrique s’écrit :

$$
V_{\mathrm{m}} \sin (\omega t-\psi)=L \frac{\mathrm{d} I(t)}{\mathrm{d} t}+R(T, t) I(t)
$$

Cette équation doit être résolue simultanément avec l'équation thermique

\section{Applications}

4.1. Caractéristiques de LA Bobine. - L'application concerne une bobine de limitation qui a été réalisée au Centre de Recherche sur Les Très Basses Températures (C.R.T.B.T.) du C.N.R.S. de Grenoble.

Ses caractéristiques sont données dans le tableau I.

Elle est utilisée comme limiteur résistif et c'est dans ces conditions que les études théoriques et expérimentales sont menées.

Le fil supraconducteur utilisé est en $\mathrm{NbTi}$ et CuNi30. Il est prévu pour un usage en régime variable (Tab. II).

Ce fil à une résistivité quasiment constante jusqu'à la température où il devient supraconducteur. Le courant critique du fil mesuré sur un échantillon court (sous champ propre) vaut 100 A $_{\text {crète. }}$

Le dispositif expérimental fait l'objet de la figure 2. Le contacteur en parallèle avec la charge est fermé pour simuler un court-circuit. Le supraconducteur transite alors par dépassement du courant critique. C'est le point de départ des simulations, le courant atteint une valeur critique en un point de la bobine. Le second contacteur, placé en série avec le limiteur, sert à ouvrir la ligne après la limitation.

4.2. Domaine D'Étude ET RÉSOlution. - Compte tenu des isolations entre couches, on pourra limiter l'étude de la propagation à une seule couche.

L'épaisseur de la bobine est faible par rapport à son rayon, ceci permet de faire l'étude en coordonnées cartésiennes en négligeant l'effet de courbure. La bobine est donc "déroulée" en une surface rectangulaire d'épaisseur négligeable. Sur les frontières matérialisées par les

Tableau I. - Caractéristiques de la bobine supraconductrice.

[Superconducting coil parameters.]

\begin{tabular}{|l|l|}
\hline $\begin{array}{r}\text { Nombre de couches } \\
\text { (couches en séne) }\end{array}$ & 2 \\
\hline $\begin{array}{r}\text { Nombre de spires } \\
\text { (par couche) }\end{array}$ & 708 \\
\hline Diamètre & $35 \mathrm{~mm}$ \\
\hline Longueur & $84 \mathrm{~mm}$ \\
\hline Inductance & $280 \mathrm{mH}$ \\
\hline Courant critique & $60 \mathrm{~A}_{\text {trrle }}$ \\
\hline
\end{tabular}


Tableau II. - Caractéristıques du fil supraconducteur [12].

[Superconducting strand parameters.]

\begin{tabular}{|l|l|}
\hline Type & monobrin \\
\hline Diamètre & $0,2 \mathrm{~mm}$ \\
\hline Nombre de filaments & 377982 \\
\hline Diamètre des filaments & $139 \mathrm{~nm}$ \\
\hline Pas de torsade & $1 \mathrm{~mm}$ \\
\hline \%NbTi & 18,7 \\
\hline \%CuNi & 81,3 \\
\hline$\% \mathrm{Cu}$ & 0 \\
\hline
\end{tabular}

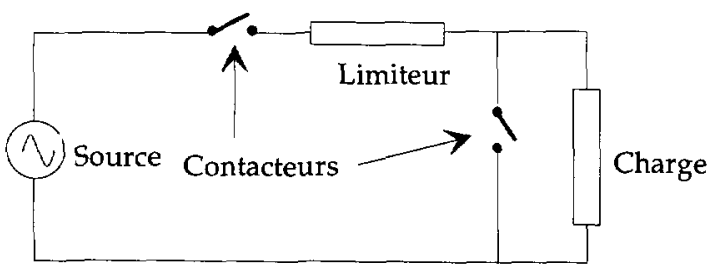

Fig. 2. - Schéma électrique de montage du limiteur.

[Electrical test scheme.]

spires d'extrémités, les conditions d'adiabaticité se traduisent par $\mathrm{d} T / \mathrm{d} x=0$, sur la frontière correspondant à la coupure il y a une condition de raccordement : $T(x, 2 \pi r, t)=T(x, 0, t)$.

Compte tenu des hypothèses, la formulation bidimensionnelle de l'équation (1) est :

$$
K_{x} h_{x} S_{y} \frac{\partial^{2} T}{\partial x^{2}}+K_{y} h_{y} S_{x} \frac{\partial^{2} T}{\partial y^{2}}-h S_{x y}\left(T-T_{\text {bain }}\right)+f \rho \frac{\delta l}{s} I^{2}=\sigma C \frac{\partial T}{\partial t}
$$

L'équation (3) est résolue par la méthode de différence finie ADI (Alternate Direction Implicit). La forte non-linéarité de tous les paramètres de l'équation (3) impose l'utilisation d'un algorithme de Newton-Raphson. Le pas de temps nécessaire pour la bonne convergence des calculs vaut $0,1 \mu \mathrm{s}$.

La résolution simultanée de l'équation électrique (2) est faite par une méthode de différence finie, un schéma totalement explicite est suffisant pour cette résolution.

4.3. RÉSUltats THÉORIQUES. - Les simulations sont initialisées après la transition et durent $100 \mu$ s. Cette durée, bien qu'insuffisante pour l'étude complète de la bobine, permet d'analyser l'influence d'un certain nombre de facteurs intervenant dans la propagation de la transition tout en gardant des temps de calcul raisonnables (quelques heures sur station de travail).

4.3.1. Influence des paramètres de construction. - Le courant critique dans une bobine est une donnée locale. Le courant critique d'une partie de conducteur est liée à sa position au sein de l'enroulement, à cause des valeurs locales de l'induction d'une part, et des contraintes 


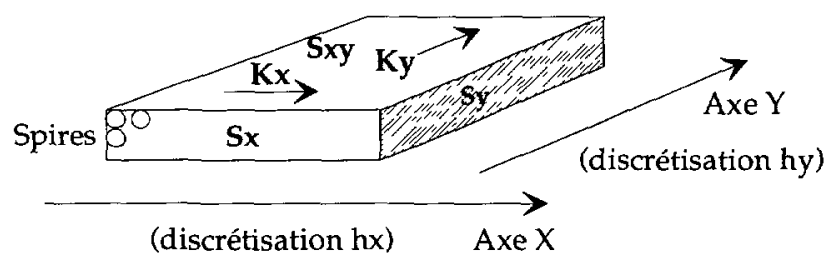

Fig. 3. - Elément d'une bobine.

[Element of coil.]

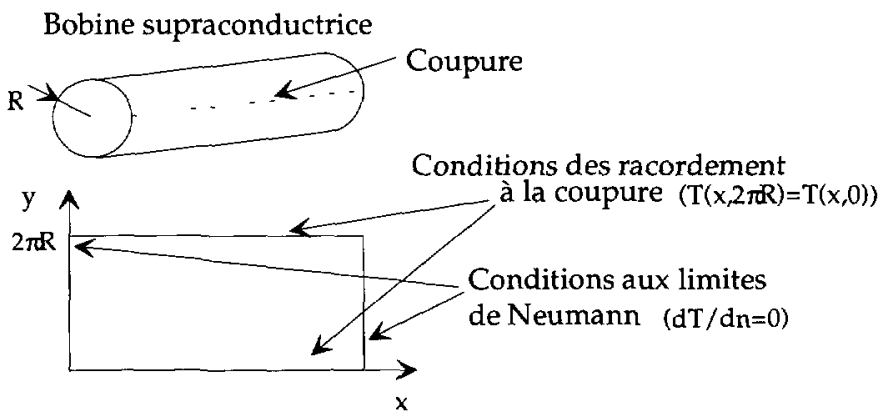

Fig. 4. - Représentation des domaines d'étude.

[Limits conditions.]

mécaniques d'autre part, ces dernières dépendent de façon importante de la qualité de fabrication (tension mécanique, imprégnation ..). On définit un rapport $I / I_{\text {co }}$, où $I_{\text {co }}$ est le courant critique du fil en échantillon court sous champ propre et $I$ le courant pour lequel la bobine transite. Dans les simulations $I$ est le courant dans le fils à l'instant de la transition et $I_{\text {co }}$ le courant critique du fil utilisé dans le calcul du "current sharing". L'évolution de la température maximale et de la vitesse de propagation en fonction du rapport $I / I_{\text {co }}$ sont calculées.

$I_{\text {lim }} / I$ est le rapport du courant $100 \mu$ s après la transition et du courant auquel la bobine a transité. Un rapport $I / I_{\text {co }}$ suffisant garantit que le courant de limitation ne dépassera pas le courant nominal (Fig. 5).

La température s'accroît fortement lorsque le courant dans la bobine à l'instant de la transition est proche de celui mesuré sur le fil en échantillon court (Fig. 6). Plus le courant initial est élevé, plus l'énergie dissipée est grande sans que les termes d'échanges et les paramètres physiques de la bobine ne varient, la température atteint une valeur de $165 \mathrm{~K}$.

Comme il vient d'être vu, la température maximale du supraconducteur croît avec le rapport $I / I_{\mathrm{co}}$, et conduit à une augmentation de la conductivité thermique et par conséquent à celle de la vitesse de propagation. Une forte vitesse de propagation peut aller de pair avec un échauffement élevé (Fig. 7).

4.3.2. Accélération de la propagation. - Les résultats précédents montrent que l'efficacité de la limitation va de pair avec une forte valeur de la température maximale atteinte par le supraconducteur. Cette valeur peut être réduite en augmentant la vitesse de propagation. Une première solution consiste à augmenter la surface de départ de la transition en utilisant une chaufferette. Une seconde solution consiste à réduire les contacts thermiques entre spires. 


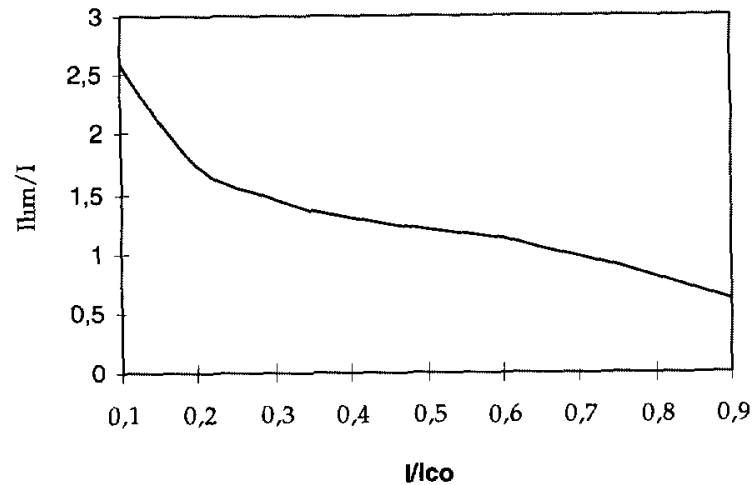

Fig. 5. - Variation du rapport $I_{1 \mathrm{~m}} / I$ avec le rapport $I / I_{\mathrm{co}}$.

[Variation of $I_{1 \mathrm{~m}} / I$ with $I / I_{\mathrm{co}}$.]

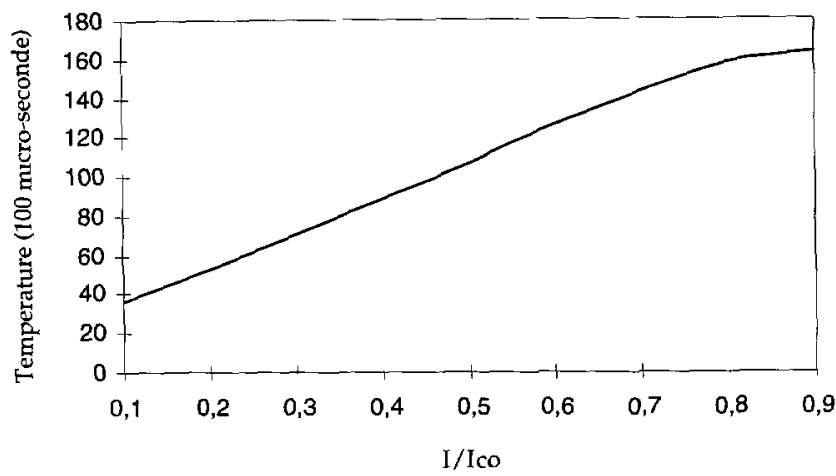

Fig. 6. - Variation de $T(100 \mu \mathrm{s})$ avec $I / I_{\mathrm{co}}$.

[Variation of $T(100 \mu \mathrm{s})$ with $I / I_{\mathrm{co}}$.]

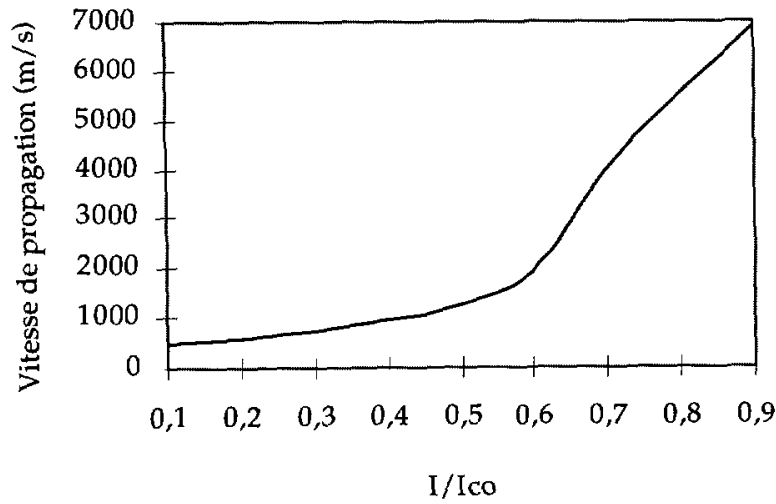

Fig. 7. - Variation de la vitesse de propagation avec le rapport $I / I_{\text {co }}$.

[Variation of propagation velocity with $I / I_{\text {co. }}$ ] 


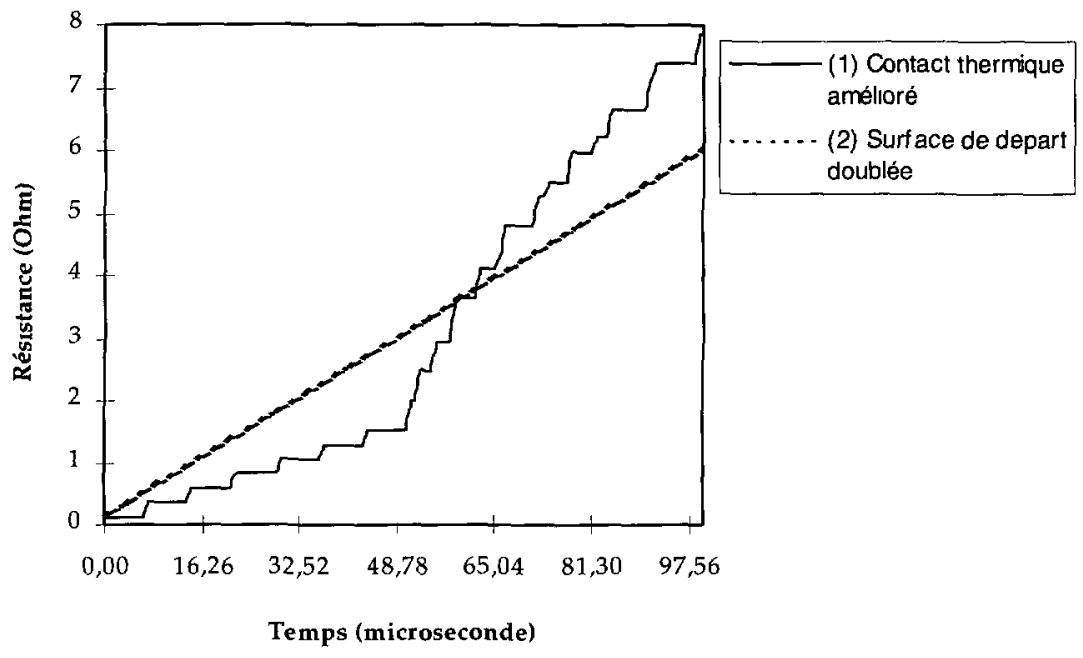

Fig. 8. - Variation de la résistance avec le temps.

[Resistance versus time.]

Les investigations suivantes sont faites avec un rapport $I / I_{\text {co }}$ de $0, \tau$ et sur une durée de $100 \mu$ s suffisante pour analyser l'influence des paramètres sans demander un temps de calcul prohibitif.

Dans un premier temps on double la surface de la zone initiale qui transite par dépassement du courant critique. Au bout de $100 \mu \mathrm{s}$, la valeur de la résistance est supérieure de $8 \%$ par rapport à celle du cas précédent. La résistance initiale est certes élevée mais la vitesse de propagation reste faible.

Une réduction de $20 \%$ du foisonnement entre spires de la même couche se traduit par une conductivité thermique latérale plus forte et permet d'augmenter la résistance de $30 \%$, la vitesse de propagation vaut maintenant $6 \mathrm{~km} / \mathrm{s}$ (Fig. 8).

4.4. RÉsultats EXPÉRIMENTAUX ET THÉORIQUes. - Le dispositif expérimental est celui de la figure 2. Un court-circuit franc aux bornes de la charge est effectué avec un déphasage de $225^{\circ}$ par rapport au passage à 0 de la tension. Le courant qui vaut, sans limiteur, $1700 \mathrm{~A}_{\text {crète }}$ n'est théoriquement plus limité que par l'impédance de source.

Les simulations sont à présent effectuées sur une durée de $5 \mathrm{~ms}$. Les résultats numériques sont comparés à ceux obtenus expérimentalement. Le rapport $I / I_{\text {co }}$ pris pour les simulations vaut 0,7 , ce paramètre pouvant être ajusté.

On observe que :

- Le courant décroît extrêmement rapidement ce qui correspond à un fonctionnement correct du limiteur. Les courbes expérimentale et théorique se superposent (Fig. 9).

- La résistance croît durant 1,8 ms avant de passer par un palier de $3 \mathrm{~ms}$. La température croît très vite dans premier temps, induisant une forte propagation puis, dans un second temps, la zone normale, qui voit sa température s'uniformiser, cesse de se propager. La résistance expérimentale est calculée à partir des mesures de courant et de tension. Les courbes expérimentale et théorique coïncident (Fig. 10). 


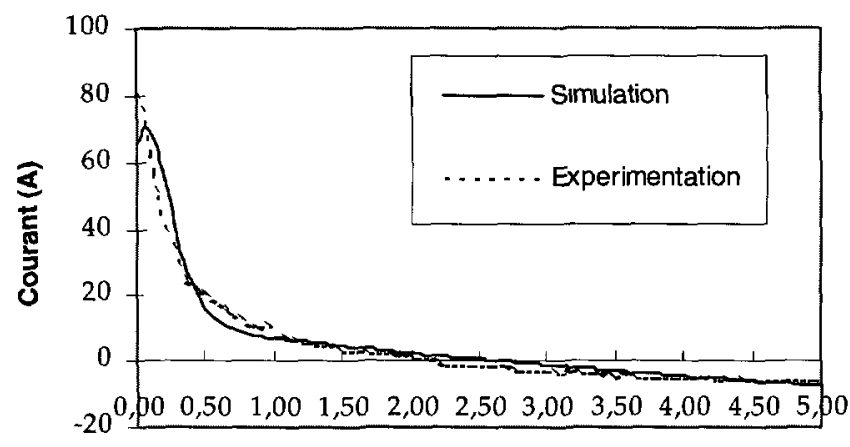

Temps (ms)

Fig. 9. - Comparaison des courants calculé et mesuré.

[Comparison between computed and measured current.]

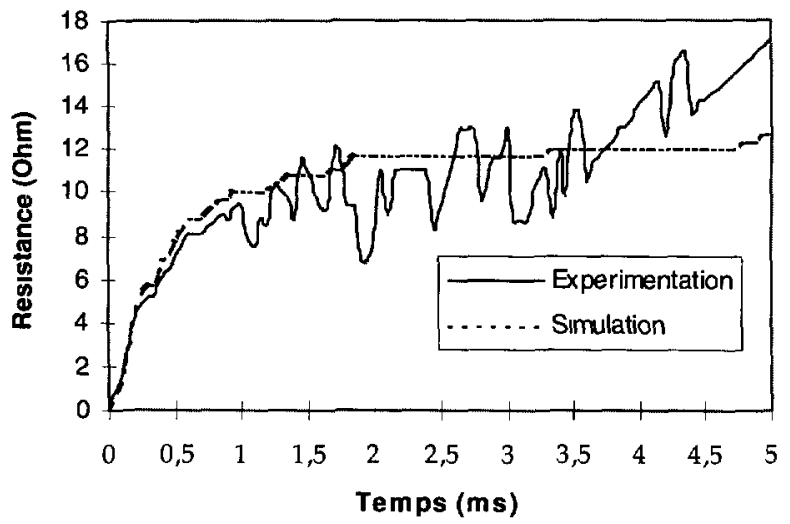

Fig. 10. - Comparaison entre les résistances calculée et mesurée.

[Comparison between computed and measured resistance.]

- La puissance maximale est dissipée durant les premiers instants de la transition (Fig. 11), c'est le moment où le supraconducteur s'échauffe le plus. Cette puissance correspond à une énergie relativement faible de 15 Joule.

- La taille de la zone normale au bout de $5 \mathrm{~ms}$ reste faible, moins de $10 \%$ de la bobine a transité comme le montre la carte de température de la figure 12 pour la totalité de la bobine. Cette distribution de température permet de calculer le courant et la résistance de la bobine. Cette propagation insuffisante est essentiellement due à un faible rapport $I / I_{\mathrm{co}}$

\section{Conclusions}

La formulation 2D d'un processus thermique est nécessaire et suffisante pour étudier la propagation qui suit la transition d'une partie d'un enroulement supraconducteur de faible épaisseur. Ce problème électrothermique a été résolu par la méthode de différence finie ADI. Les résultats 


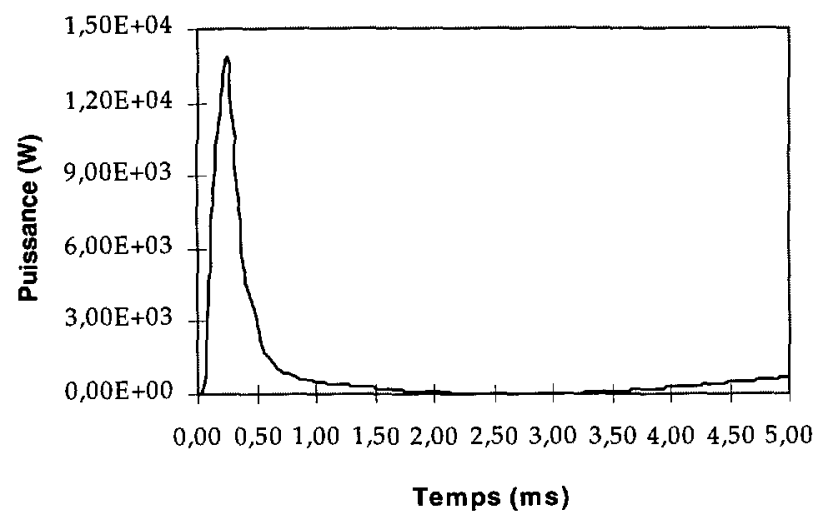

Fig. 11. - Puissance dissipée (simulation).

[Dissipated power, simulation.]

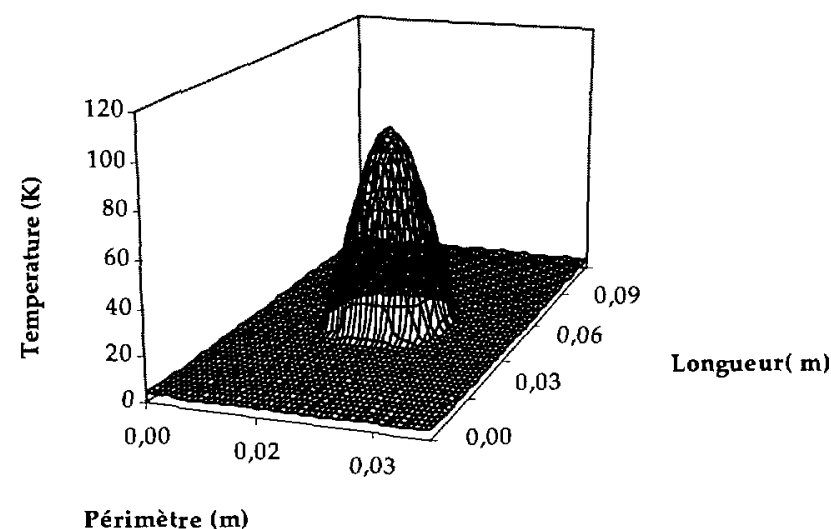

Fig. 12. - Distribution de température $5 \mathrm{~ms}$ après la transition.

[Temperature distribution $5 \mathrm{~ms}$ after quench.]

des simulations sont tout à fait cohérents avec les résultats expérimentaux. L'influence de la taille de la zone normale initiale, des facteurs liés à la réalisation de la bobine ont été étudiés séparément; ils ont chacun une influence non négligeable sur la température atteinte par le supraconducteur et la propagation de la transition.

\section{Remerciements}

Les auteurs tiennent à remercier Messieurs P. Tixador avec qui la partie expérimentale fut menée et $A$. Boulbes pour la réalisation du bobinage supraconducteur. 


\section{Bibliographie}

[1] Berglund R.O., Mittelstadt W.A., Shelton M.L., Barkan P., Dewey C.G. et Skreine K.M., One cycle interruption at $500 \mathrm{kV}$; system benefits and breaker design, IEEE Trans. Power Appar Syst 93 (1974) 1240-1251.

[2] Slade P.G., Wu J.L., Stacey E.J., Stubler W.F., Voshall R.E., Bonk J.J., Porter J. et Hong L., The utility requirement for a distribution fault current limiter, IEEE Trans. Pow. Deliv. 7 (1992) 507-515.

[3] Grey K.E. et Fowler D.E., A superconducting fault-current limiter, J. Appl. Phys. 49 (1978) 2546-2550.

[4] Verhaege T., Cottevieille C., Weber W., Thomas P., Therond P.G., Laumond Y., Bekhaled M. et Pham V.D., Progress on superconducting current limitation project for the french electrical grid, IEEE Trans. mag. 30 (1994) 1907-1910.

[5] Ito D., Tsurunaga K., Tada T., Yoneda E.S., Hara T., Okinawa K., Okhuma K. et Yamamoto T., Development of $6.6 \mathrm{kV} / 1.5 \mathrm{kA}$-class superconducting fault current limiter, Cryog. 32 (1992) $462-465$.

[6] Tixador P., Lévêque J., Brunet Y. et Pham V.D., Hybrid AC superconducting current limiter: small scale experimental model, IEE Proc. Gener. Distrib. 141 (1994) 117-124.

[7] Tixador P., Lévêque J., Brunet Y. et Pham V.D., Hybride superconducting a.c. current limiter extrapolation 63 kV-1250 A, J. Phys. III France 4 (1994) 603-614.

[8] Wilson M.N., Superconducting magnet, Oxford science publication.

[9] Yoneda E.S. et Ito D., Very apparent normal zone propagation velocity in ac superconducting coil, Proc. MT 112 (Tsukuba, 1989) 1096-1101.

[10] Dresner L., Propagation of normal zones in composite superconductors, Cryog. 16 (1976) 675-681.

[11] Maddock B.J., James G.B. et Norris W.T., Superconducting composites : heat ransfert and steady state stabilization, Cryog. 9 (1969) 261-273.

[12] GEC-ALSTHOM, av, des trois chênes, 90000 Belfort. 